> La répression catabolique permet aux bactéries, mais aussi aux levures ou champignons, une utilisation préférentielle des sources de carbone. Ce phénomène se traduit par une croissance diauxique ${ }^{1}$ durant laquelle les bactéries assimilent d'abord les sources de carbone rapidement métabolisables, puis les sources de carbone non préférentielles. Divers mécanismes moléculaires sont responsables de la répression catabolique et contrôlent non seulement l'expression de gènes impliqués dans l'utilisation de sources de carbone alternatives, mais aussi l'expression de plusieurs gènes impliqués dans des processus cellulaires variés. Cette synthèse décrit les principaux mécanismes moléculaires retrouvés chez les entérobactéries et chez les firmicutes, ainsi que l'importance du système des phosphotransférases dans cette régulation. <

La répression catabolique (RC) est un phénomène mis en place lorsque des bactéries, mais aussi des levures ou des champignons, sont cultivées en présence de deux (voire plusieurs) sources de carbone. Appelée aussi « effet glucose », elle est l'un des mécanismes les plus conservés chez les microorganismes. Elle les protège d'un gaspillage d'énergie en leur évitant de synthétiser des protéines superflues dans des conditions données. Chez les bactéries, cela se traduit par une courbe de croissance biphasique (Figure 1). Ce phénomène, mis en évidence par Jacques Monod en 1942, s'appelle diauxie [1]. Après une première phase de croissance exponentielle, due à l'utilisation par les bactéries de I'hydrate de carbone le plus rapidement métabolisable (généralement le glucose, mais pas toujours [2]), la croissance s'arrête puis reprend à nouveau, de façon exponentielle, grâce à l'utilisation d'une autre source de carbone présente dans le milieu de culture. Cette utilisation hiérarchique des hydrates de carbone est

Vignette (Photo @ Inserm - Émilie Cloup et Jean-Philippe Nougayrede)

${ }^{1}$ Courbe de croissance biphasique avec métabolisme d'une source de carbone, puis d'une autre.

\section{La répression catabolique ou comment les bactéries choisissent leurs sucres préférés}

Anne Galinier

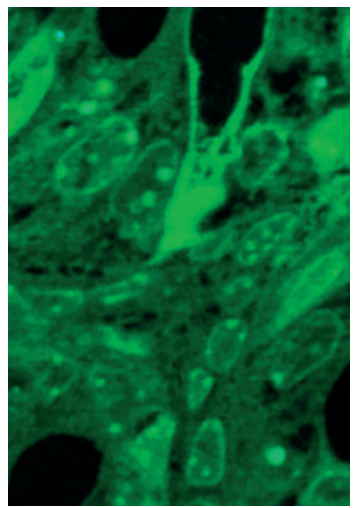

Laboratoire de chimie bactérienne, UMR 7283, CNRS - Aix Marseille Univ, Institut de Microbiologie de la Méditerranée (IMM), 31, chemin Joseph Aiguier, 13402, Marseille, Cedex 20,

France.

galinier@imm.cnrs.fr

due à l'inhibition de la synthèse des enzymes impliquées dans le catabolisme des sources de carbone non-préférentielles, d'où le nom de répression catabolique. La latence entre les deux phases de croissance exponentielle s'explique par le temps indispensable à la bactérie pour synthétiser les enzymes nécessaires à l'utilisation de la source de carbone non-préférentielle, lorsque la RC est levée (Figure 1).

Or, la RC est un mécanisme de régulation global ayant un impact majeur sur la physiologie bactérienne. Non seulement elle intervient dans l'inhibition de l'expression de gènes impliqués dans le transport et le métabolisme des sources de carbone non préférentielles, mais elle affecte aussi l'expression de nombreux gènes impliqués dans des processus variés. En particulier, il a clairement été établi, chez plusieurs espèces bactériennes pathogènes tels que Staphylococcus aureus ou Listeria monocytogenes, que RC et virulence étaient étroitement imbriquées [3]. Chez Bacillus subtilis, l'expression de $10 \%$ des gènes est régulée par le glucose [4]. Ce phénomène de RC prend particulièrement de l'importance lorsqu'il y a compétition entre plusieurs microorganismes dans des milieux naturels, puisque l'utilisation de sources de carbone préférentielles est un facteur déterminant dans le taux de croissance bactérienne [5]. De fait, la RC a des implications en biotechnologie comme les biotransformations, l'utilisation d'usines bactériennes et la bioremédiation, etc. [6] et, pour certaines applications industrielles, il est parfois nécessaire de s'affranchir de la RC pour optimiser la production [7].

Le phénomène de RC, initialement décrit pour B. subtilis [1], a fait I'objet d'études approfondies chez plusieurs bactéries. II a été d'abord bien caractérisé chez Escherichia coli [8]. Chez ces deux bactéries 


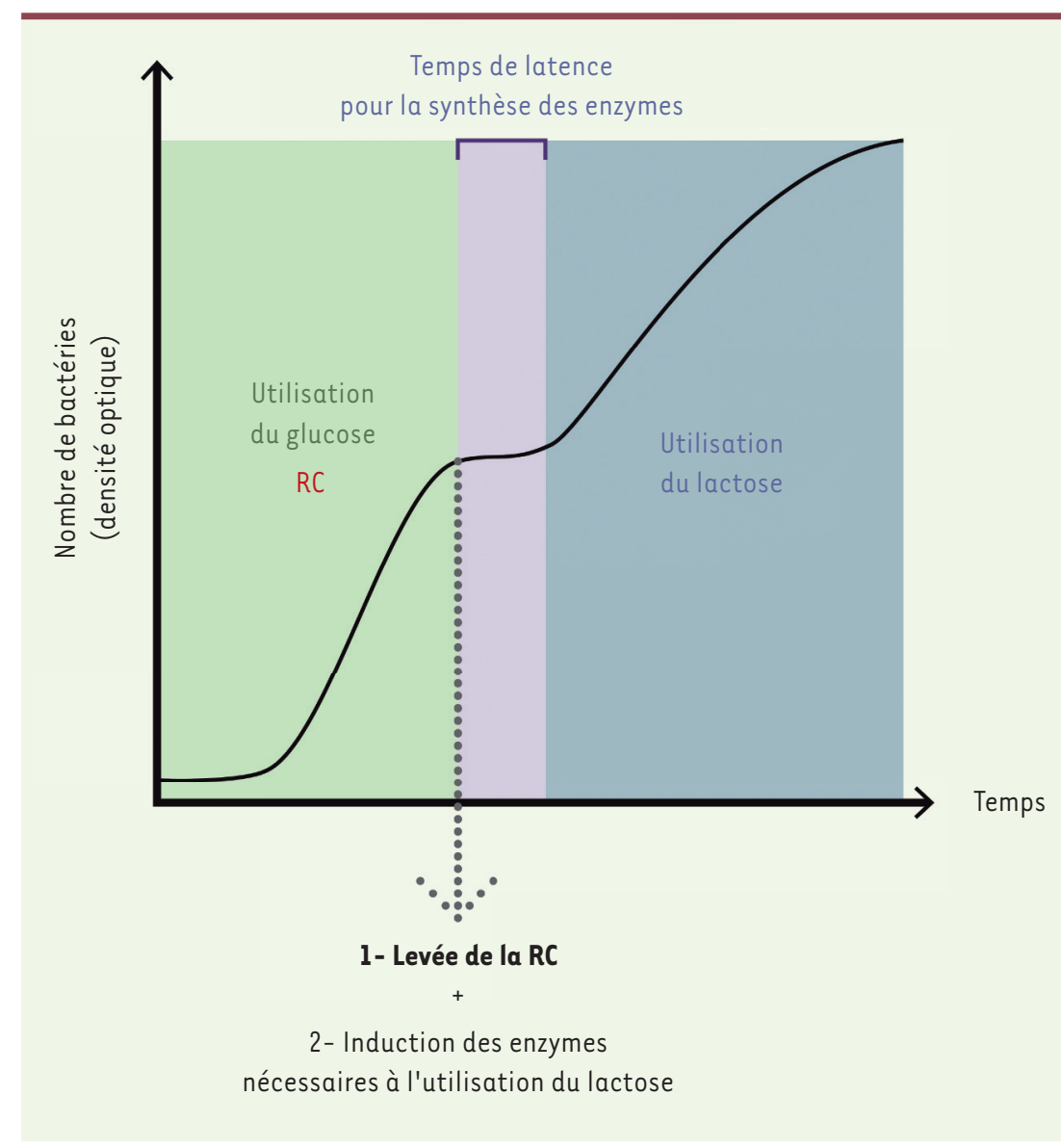

Figure 1. Le phénomène de diauxie. Lorsque des bactéries, telles que $\varepsilon$. coli, sont cultivées sur milieu contenant deux sucres comme le glucose et le lactose, elles utilisent en priorité le glucose. Celui-ci est métabolisé par des enzymes synthétisées de façon constitutive alors que la synthèse des enzymes nécessaires à l'utilisation du lactose est réprimée (phénomène de répression catabolique ou RC). Quand le glucose est épuisé, la RC est abolie ; les bactéries peuvent induire spécifiquement la synthèse des enzymes impliquées dans la dégradation du sucre présent, le lactose, qui joue le rôle d'inducteur.

phosphorylation concomitante d'un sucrePTS est l'autophosphorylation d' $\varepsilon l$ à partir d'un donneur de phosphate très riche en énergie, le phosphoénolpyruvate (ou PEP) [11]. L'El phosphorylée ( $P \sim \varepsilon \mid$ ) transfère ensuite son groupement phosphate à HPr. P HPr transmet à son tour son phosphate à une EllA, qui phosphoryle l'EllB correspondante. Tous ces phosphotransferts à haute énergie sont réversibles et se produisent au niveau d'histidines, à l'exception des protéines EIIB pour lesquelles, habituellement, c'est une cystéine qui est modèles, il est principalement contrôlé par le niveau de phosphorylation de protéines impliquées dans le transport d'hydrates de carbone et appartenant au système des phosphotransférases (PTS).

L'objectif de cette synthèse est de décrire les mécanismes les mieux caractérisés impliqués dans la RC, et d'explorer l'importance du système des phosphotransférases dans ce phénomène de régulation.

\section{Le transport des hydrates de carbone par le PTS}

Le système des phosphotransférases (PTS), découvert il y a plus de 50 ans $[9,10]$, transporte et phosphoryle de façon concomitante un certain nombre d'hydrates de carbone chez de nombreuses bactéries et certaines archaea. Il est généralement composé de plusieurs phosphotransférases : l'enzyme I ( $(\varepsilon)$ ), la protéine $\mathrm{HPr}$ (histidine-containing protein), et plusieurs complexes multi-protéiques formant les enzymes $\|(\varepsilon \|)$. Les protéines $\varepsilon l$ et $H \operatorname{Pr}$ sont solubles et impliquées dans l'incorporation de la plupart des substrats du PTS. En revanche, les $\varepsilon$ Il, constituées de protéines cytoplasmiques ( $\varepsilon$ IIA et $\varepsilon \| B$ ) et transmembranaires (EIIC et éventuellement $\varepsilon I I D$ ), sont habituellement spécifiques d'un substrat donné ou d'un petit groupe de sucres étroitement apparentés (Figure 2). Les protéines $\varepsilon$ l et HPr sont généralement synthétisées de façon constitutive alors que la synthèse des complexes $\varepsilon \|$ est induite par la présence du sucre transporté par le PTS (sucre-PTS) dans le milieu extracellulaire. La première étape du transport et de la phosphorylée. À la dernière étape, P EIIB transfère son groupement phosphate à l'hydrate de carbone complexé et transporté par l'EIIC. Dans des conditions physiologiques, cette réaction est pratiquement irréversible, la phosphorylation de l'hydrate de carbone diminuant probablement l'affinité de ce dernier pour l'ElIC afin d'être libéré dans le cytoplasme (Figure 2).

Chez les firmicutes, la protéine HPr est phosphorylée non seulement au niveau de l'histidine en position 15 (His-15), à partir de PEP et par $\varepsilon 1$, mais également au niveau de la sérine en position 46 (Ser-46), à partir d'ATP et par I'HPr kinase/phosphorylase (HPrK/P) [12, 13]. La phosphorylation d'HPr à partir d'ATP n'est pas impliquée dans l'incorporation et la phosphorylation des sucres, mais a des fonctions régulatrices, en particulier dans la RC [14]. Les entérobactéries telles qu' $\varepsilon$. coli ne possèdent pas d'HprK/P et la région autour de la Ser-46 d'HPr n'est pas conservée.

\section{La RC chez $\varepsilon$. coli et chez Enterobacteriaceae : un phénomène bien caractérisé}

Chez $\varepsilon$. coli et chez les Enterobacteriaceae, l'un des acteurs majeurs de la RC est I'EIIA $A^{G l c}$, un constituant 


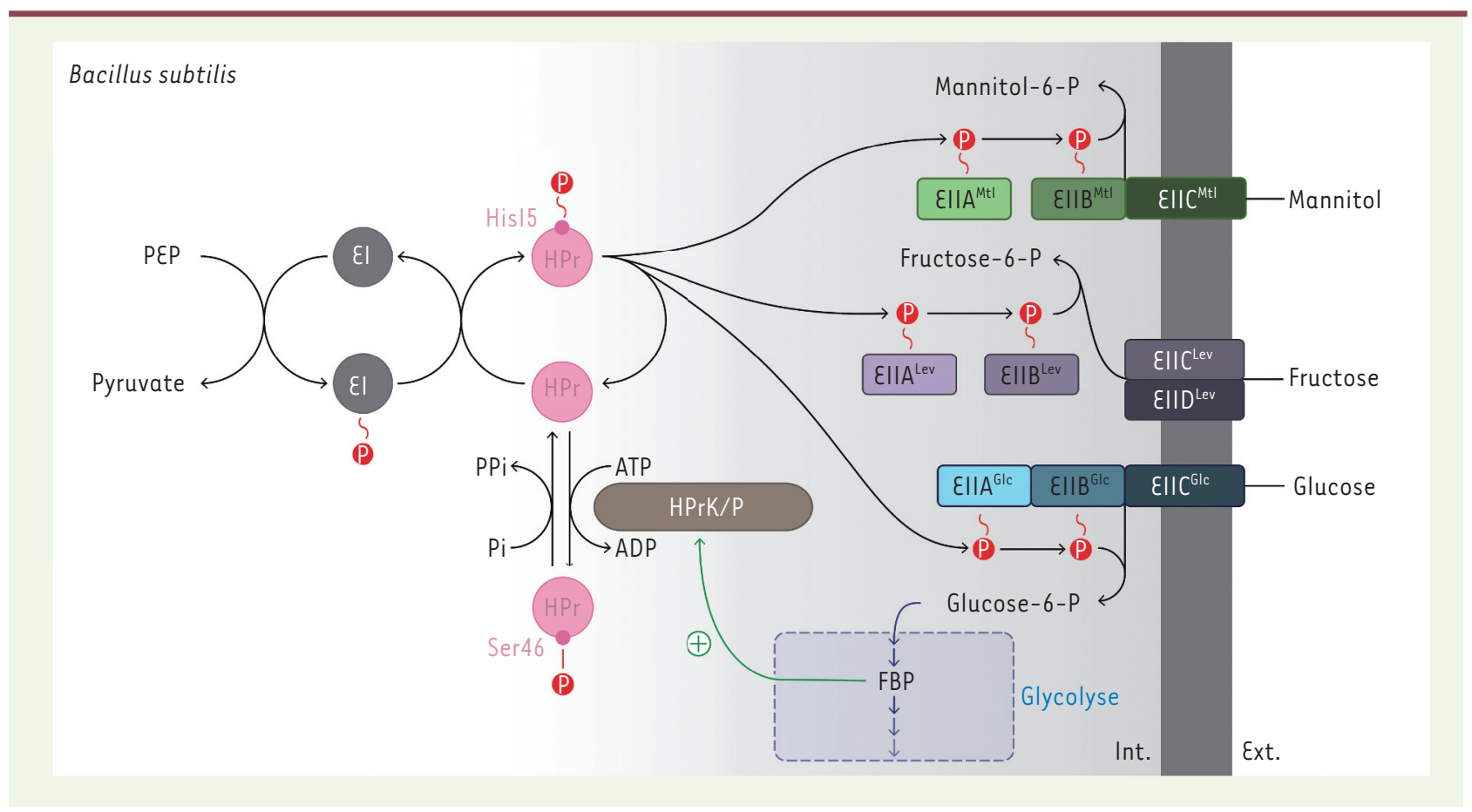

Figure 2. Exemple de trois systèmes des phosphotransférases (PTS) chez B. subtilis. Le PTS est généralement composé des protéines solubles Enzyme I ( $(\varepsilon)$ et $\operatorname{HPr}$ (histidine-containing protein) qui sont impliquées dans l'incorporation de la plupart des substrats PTS, alors que les Enzymes II (દાI) sont spécifiques d'un substrat ou d'un petit groupe de sucres donnés. Pour indiquer la spécificité des $\varepsilon \| l$, une abréviation de trois lettres du substrat transporté est ajoutée en exposant au domaine $\varepsilon \| l$ correspondant. Ces $\varepsilon \| l$ sont constitués de protéines cytoplasmiques ( $\varepsilon$ IIA et $\varepsilon I I B)$ et d'une, voire deux, protéines transmembranaires ( $દ ા I C$ et $\varepsilon I I D)$. Le transport et la phosphorylation concomitante des sucres-PTS sont réalisés via des réactions de phosphotransferts, le donneur de phosphate étant le phosphoénolpyruvate (ou PEP). À la dernière étape, P-ElIB transfère son groupe phosphate à l'hydrate de carbone complexé et transporté par l'ElIC ou l'ElICD. Chez les firmicutes, la protéine HPr est non seulement phosphorylée au niveau de l'histidine His-15 à partir de PEP et par EI, mais également au niveau de la sérine Ser-46 à partir d'ATP par l'HPr kinase/ phosphorylase (HPrK/P) [12,13], lorsque la concentration en fructose 1,6 bisphosphate (FBP) intracellulaire est élevée.

de l'Ell impliquée dans le transport du glucose. Plus précisément, c'est l'état de phosphorylation de l'ElIA ${ }^{\text {Glc }}$ qui contrôle la RC; celui-ci semble être principalement déterminé par le rapport des concentrations intracellulaires de PEP et de pyruvate. En effet, lorsqu'un sucre rapidement métabolisable tel que le glucose est disponible, le rapport $[P E P] /[p y r u v a t e]$ est faible, et l'EllA Glc est présente principalement sous sa forme non phosphorylée [15]. Ainsi, elle ne peut stimuler l'adénylate cyclase et/ou inhiber l'AMPc phosphodiestérase [16]. La concentration d'AMPc intracellulaire est donc faible. Le régulateur transcriptionnel CRP ( $C A M P$ receptor protein, également connu sous le nom de CAP [catabolite activator protein]), qui doit interagir avec I'AMPc pour pouvoir se lier à l'ADN, ne peut alors pas reconnaître ses séquences d'ADN cibles [17]; l'expression des opérons sensibles à la RC n'est donc pas induite (Figure 3). En revanche, en présence de sucres qui ne sont pas transportés par le PTS, ou des sucres-PTS lentement métabolisables, I'ElIA $A^{G l c}$ est présente principalement sous sa forme phosphorylée et peut ainsi stimuler la synthèse d'AMPc [15]. Le complexe AMPc/CRP peut se lier à ses séquences promotrices spécifiques, augmenter l'affinité de l'ARN polymérase pour les promoteurs, et ainsi induire l'expression des opérons sensibles à la $R C$ en présence d'un inducteur. Par exemple, lorsque du glucose et du lactose sont tous deux présents dans le milieu de culture, le glucose doit être consommé pour permettre la liaison du complexe AMPc/CRP à l'ADN, tandis que le lactose intracellulaire est nécessaire pour décrocher le répresseur lactose de sa séquence opératrice. Lorsque ces deux conditions sont remplies, la transcription des gènes dépendant du lactose est alors induite [18]. La RC est donc due à l'absence d'activation transcriptionnelle.

Des données récentes concernant des Vibrionaceae, qui, comme $\varepsilon$. coli appartiennent aux $\gamma$-protéobactéries, semblent modifier un peu ce dogme [19]. En effet, il semblerait que le complexe AMPc-CRP de Vibrionaceae joue son rôle métabolique habituel. Cependant, les variations du taux d'AMPc ne paraissent pas dépendre de la régulation de l'activité de l'adénylate cyclase par $\varepsilon \| A^{G l c}$, mais elles seraient dues à l'export et à la dégra- 


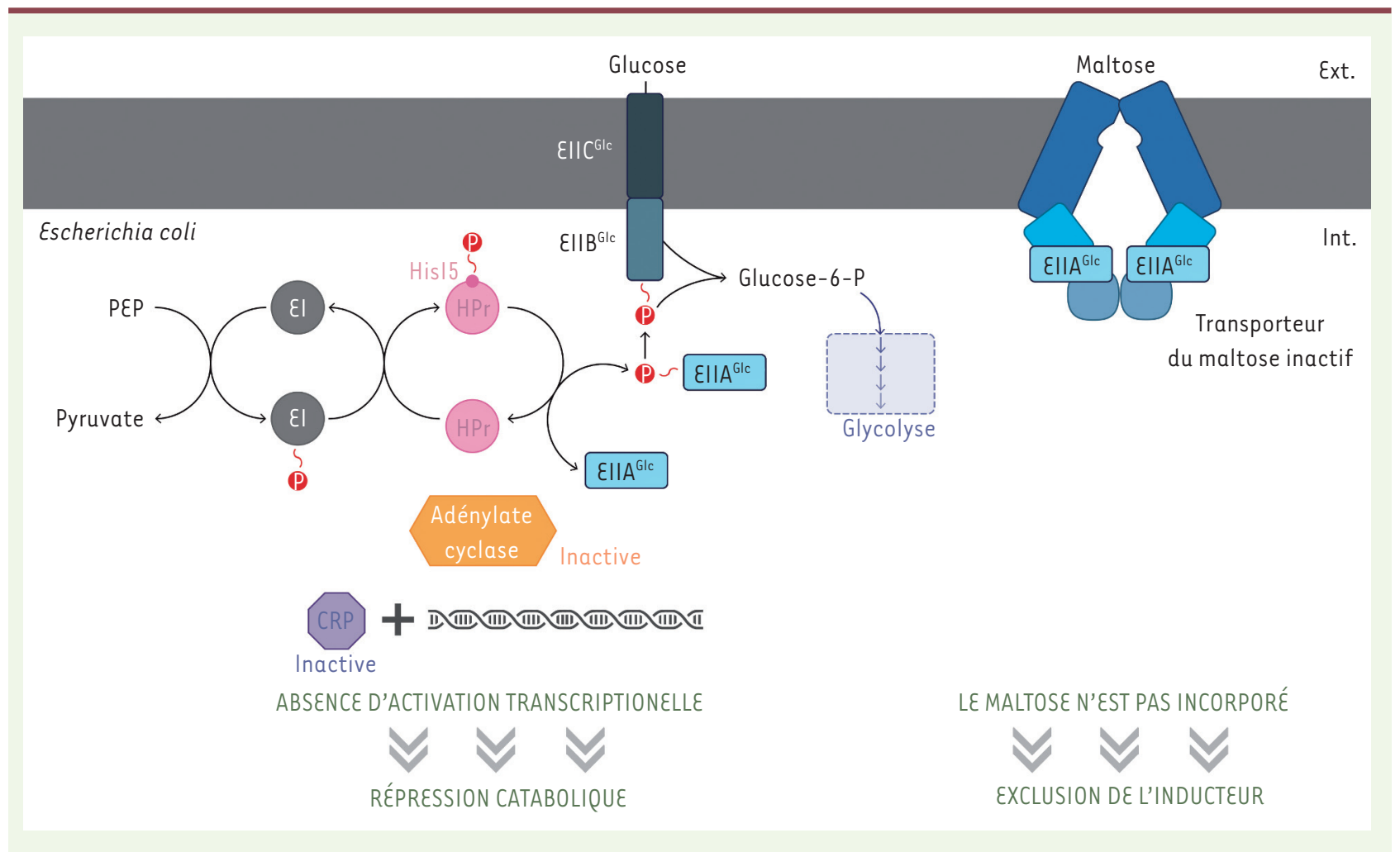

Figure 3. La RC et l'exclusion de l'inducteur chez $\varepsilon$. coli. Lorsqu'un sucre rapidement métabolisable tel que le glucose est disponible, l'ElIA ${ }^{G l c}$ est présente principalement sous forme non phosphorylée. Elle ne peut stimuler l'adénylate cyclase et/ou inhiber l'AMPc phosphodiestérase [16]. La concentration d'AMPc intracellulaire est donc faible. Le régulateur transcriptionnel CRP (cAMP receptor protein, également connu sous le nom de CAP [catabolite activator protein]), qui doit interagir avec l'AMPc pour pouvoir se lier à l'ADN, ne peut reconnaître ses séquences d'ADN cibles ; l'expression des opérons sensibles à la répression catabolique (RC) n'est pas induite. De plus, l'ElIAGlc non phosphorylée peut interagir avec MalK, la protéine fixant les nucléotides (NBP) de l'ABC transporteur du maltose, et inhiber ainsi le transport de cet hydrate de carbone. Le maltose n'étant plus incorporé à l'intérieur de la bactérie, il ne va pas y avoir induction des gènes impliqués dans l'utilisation de ce sucre. C'est le phénomène d'exclusion de l'inducteur.

dation de l'AMPc. De plus, bien que la protéine CRP nécessite d'être complexée à l'AMPc pour interagir avec ses séquences d'ADN cibles, son activité peut être régulée en réponse au glucose par un mécanisme inconnu indépendant de l'AMPc intracellulaire. Ces observations inattendues nous amènent à reconsidérer les rôles cellulaires de la CRP et de l'AMPc, et d'élargir notre vision de la RC avec des spécificités qui dépendent probablement des espèces bactériennes [19].

\section{La RC chez les firmicutes : un mécanisme moléculaire souvent méconnu}

Pendant de nombreuses années, le mécanisme moléculaire de la RC chez les Enterobacteriaceae, découvert dans les années 1970 et fondé sur l'absence d'activation transcriptionnelle des gènes cataboliques par le complexe AMPc/CRP, a été considéré comme universel. Cependant, il existe des bactéries Gram-positives ne synthétisant pas d'AMPc, et un mécanisme de RC différent a été découvert vingt ans plus tard chez $B$. subtilis [20-22]. Chez cette bactérie et chez d'autres firmicutes, un autre composant du PTS joue un rôle central dans la RC. II s'agit de la protéine HPr et plus précisément de son état de phosphorylation (Figure 4). En effet, en plus de sa phosphorylation de l'His-15 en présence d'un sucrePTS lors des phosphotransferts décrits précédemment, la protéine HPr peut également être phosphorylée sur la Ser-46 à partir d'ATP. Toutefois, selon les espèces bactériennes, ces deux phosphorylations ne sont pas toujours compatibles in vivo. Lorsqu'un sucre rapidement métabolisable est disponible, tel que le glucose ou le fructose, la glycolyse est active et le taux de fructose 1,6 bis-phosphate (FBP) est élevé. Ce métabolite est capable d'interagir avec I'HPrK/P afin de stimuler fortement son activité kinase [12, 23]. L'HPrK/P est une enzyme bifonctionnelle atypique qui catalyse la phosphorylation d'HPr sur son résidu Ser-46 ainsi que sa déphosphorylation [24]. 


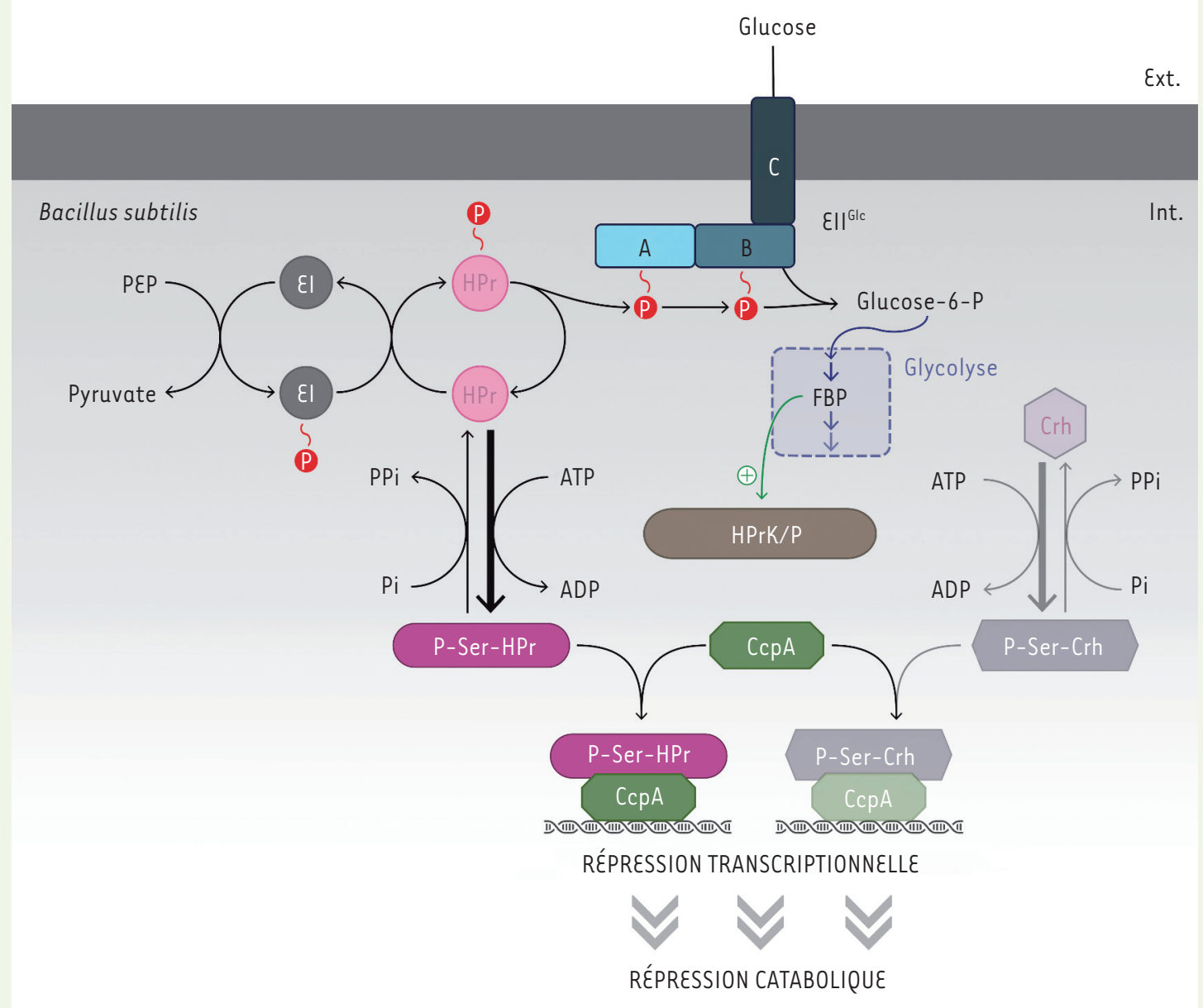

Figure 4. La RC chez B. subtilis. L'incorporation de glucose (ou de fructose) conduit à une augmentation de la concentration intracellulaire de fructose 1,6 bis-phosphate (FBP). Ce métabolite stimule l'activité kinase de l'HPr (histidine-containing protein) kinase/phosphorylase (HPrk/P) et donc la phosphorylation ATP-dépendante d'HPr et de Crh (catabolite repression HPr) au niveau du résidu Ser-46. Seules les formes séryl-phosphorylées de HPr et Crh peuvent interagir avec CcpA (catabolite control protein A). Le complexe P-Ser-HPr/CcpA ou P-Ser-Crh/CcpA se lie aux séquences cre (catabolite-responsive element) sur l'ADN et réprime généralement l'expression des gènes soumis à la RC.

Lorsque la concentration intracellulaire de FBP est élevée, $\mathrm{HPr}$ est présente principalement sous sa forme séryl-phosphorylée et peut ainsi interagir avec le régulateur transcriptionnel CcpA (catabolite control protein A) [20]. Le complexe protéique P-Ser-HPr/CcpA peut ainsi se lier efficacement à ses sites opérateurs spécifiques, appelés cre (catabolite-responsive element), afin d'inhiber l'expression des opérons sensibles à la RC [8]. En effet, dans la plupart des cas, CcpA seul ne se lie pas bien aux séquences cre et ne peut réprimer l'expression des opérons sensibles à la RC (Figure 4). Ces séquences cre sont des semi-palindromes fortement dégénérés, avec une séquence consensus mal conservée [25]. Une analyse bioinformatique indique que le génome de $B$. subtilis possèderait 126 sites cre (certains connus, d'autres putatifs) [26], et une analyse transcriptomique d'un mutant ccpA cultivé en présence ou en l'absence de glucose, a montré que $10 \%$ des gènes de $B$. subtilis étaient régulés par CcpA et soumis à la RC [4]. Ceci indique que la RC est un mécanisme de régulation globale affectant de nombreux gènes et opérons, certains n'étant pas impliqués dans le métabolisme du carbone. L'affinité du complexe P-Ser-HPr/CcpA pour ses cibles ADN dépend des caractéristiques des cre comme leur localisation par rapport au site d'initiation de la transcription, ou la position spécifique de certaines bases [27]. CcpA agit principalement comme un répresseur, mais fonctionne parfois aussi comme un activateur selon la localisation des cre. En effet, lorsque ceux-ci chevauchent le promoteur, le complexe $\mathrm{P}$-Ser-HPr/CcpA a un rôle répresseur en empêchant la liaison de la machinerie de transcription au promoteur (c'est le cas le plus fréquent). Lorsque les cre sont situées en amont du pro- 


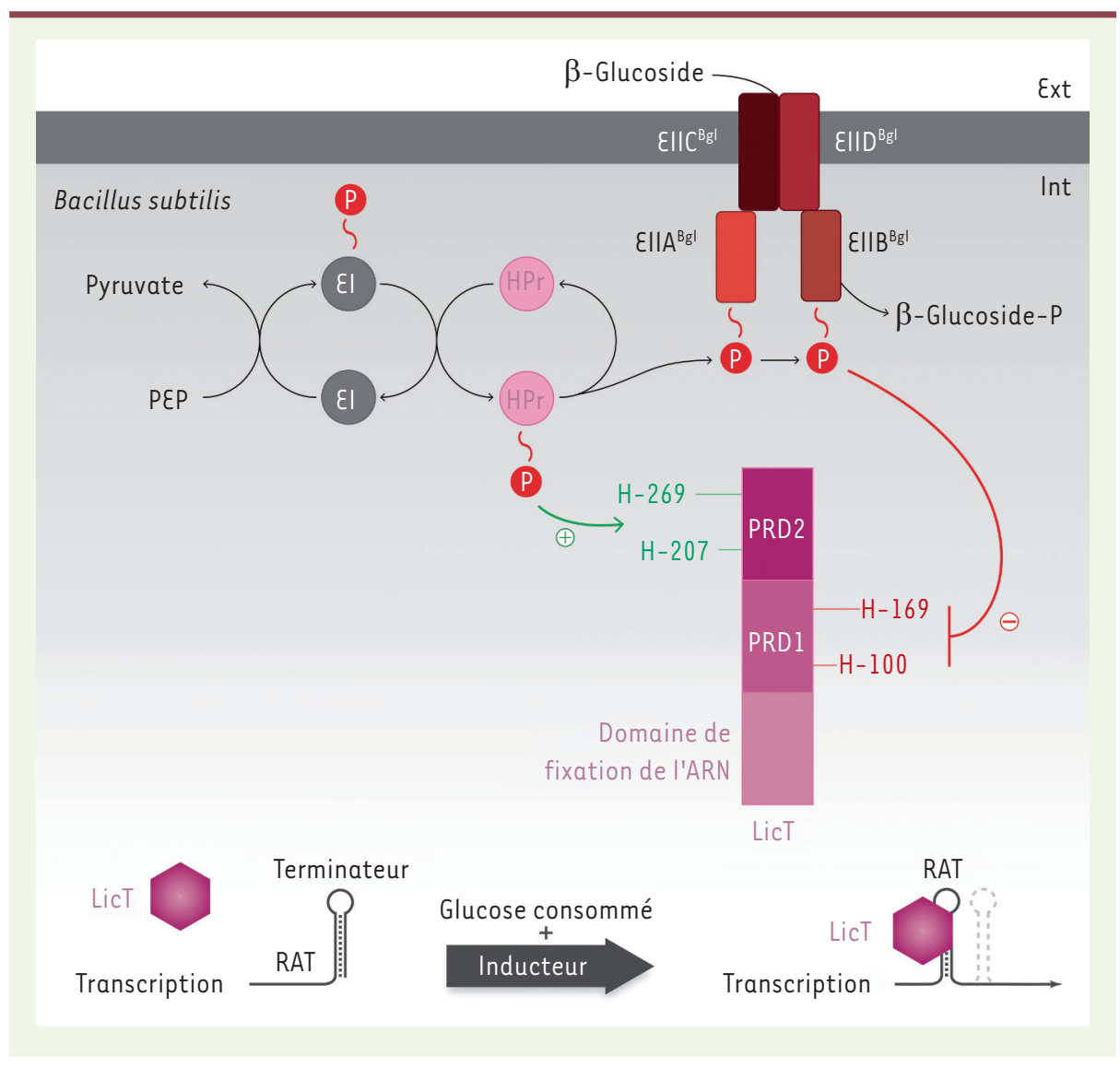

Figure 5. Régulation des régulateurs transcriptionnels contenant des domaines protéiques appelés PRD : cas de l'antiterminateur LicT de B. subtilis. La protéine LicT est un antiterminateur transcriptionnel qui régule l'expression de l'opéron bgl impliqué dans le métabolisme des $\beta$-glucosides chez $B$ subtilis. II contient deux PRD (PTS regulation domain), constitués d'environ 75 acides aminés. Chacun de ces PRD possède deux histidines phosphorylables conservées. La phosphorylation des histidines du PRDl, catalysée par $P-\varepsilon I I B^{B g I}$, inhibe l'activité de LicT alors que la phosphorylation des histidines du PRD2, catalysée par P-His-HPr, stimule la fixation de LicT à I'ARN. En présence de glucose, PRD2 n'est pas phosphorylée car il y a peu de P-His-HPr : LicT est inactif. De même, en l'absence de $\beta$-glucosides, il y a phosphorylation au niveau du PRDl : LicT est donc également inactif. En revanche, lorsque des $\beta$-glucosides sont présents dans le milieu de culture en l'absence d'un sucre-PTS répresseur, ces $\beta$-glucosides sont transportés et phosphorylés préférentiellement par P-EllB Bgl. Dans ces conditions, absence de glucose (ou d'un sucre répresseur) et présence de $\beta$-glucosides, les histidines du PRDl ne sont pas phosphorylées; celles du PRD2 le sont par P His-HPr. LicT se fixe sur l'ARN au niveau de la région RAT (antiterminateur ribonucléique) et empêche la formation d'un terminateur ; il y a donc transcription de la totalité de l'opéron bgl.

moteur, le complexe P-Ser-HPr/CcpA a, en revanche, un effet activateur. Ainsi, chez les firmicutes, le mécanisme moléculaire de RC ne repose pas sur l'absence d'activation transcriptionnelle comme chez les Enterobacteriaceae, mais est généralement dû à une répression active des gènes cataboliques. Comme chez les Enterobacteriaceae, la présence de l'inducteur dans la cellule (à savoir, la source de carbone moins favorable) est nécessaire pour la transcription des gènes correspondants.

Certains bacilles et certaines clostridies possèdent une protéine supplémentaire de type HPr, appelée Crh (catabolite repression HPr) [28]. Elle possède la Ser-46 et est phosphorylée à partir d'ATP par l'HPrK/P. $\varepsilon n$ revanche, elle est dépourvue d'His-15, le site de phosphorylation PEP-dépendant, et n'est donc pas phosphorylée par le PEP et I'El. Elle n'est, ainsi, pas impliquée dans le transport des sucres-PTS, mais possède des fonctions régulatrices. Son rôle régulateur principal est le contrôle de la formation de méthylglyoxal, un composé issu du glycéraldéhyde-3-phosphate et de la dihydroxyacétone phosphate, intermédiaires de la glycolyse [29]. Crh phosphorylée sur sa Ser-46 (P-Ser$\mathrm{Crh}$ ) est également impliquée dans la RC, mais à un moindre niveau que la P-Ser-HPr (Figure 4). En effet, l'inactivation de Crh seul n'a aucun effet mais, chez des mutants dans lesquels la phosphorylation d'HPr dépendante de I'ATP a été abolie (par remplacement de la Ser-46 par une Alanine), certains opérons cataboliques sont encore partiellement réprimés, alors qu'ils sont complètement déréprimés chez des mutants dont le gène $\operatorname{ccp} A$ a été délété [28]. La RC résiduelle observée dans les mutants du gène codant HPr disparaît quand le gène crh est également délété, ou lorsque le site de phosphorylation de la protéine Crh est inactivé $[30,31]$.

\section{Des mécanismes de $\mathrm{RC}$ retrouvés chez des firmicutes et des entérobactéries}

D'autres mécanismes moléculaires impliqués dans le contrôle de la RC et dans lesquels le PTS joue un rôle clé sont connus. Dans cette revue, nous en présenterons deux. 
Régulation PTS-dépendante de facteurs transcriptionnels contenant des «PRD »

Un de ces mécanismes de RC, retrouvés aussi bien chez des entérobactéries que chez des firmicutes, fait intervenir des facteurs transcriptionnels particuliers qui contiennent des domaines protéiques appelés PRD (PTS regulation domain) [32]. Ces domaines sont la cible de régulations directes par des composants du PTS, à savoir P-His$\mathrm{HPr}$, ainsi que des P-EIIA ou P-EIIB (Figure 5). Ces anti-terminateurs ou activateurs transcriptionnels régulent un petit nombre de gènes ou d'opérons codant généralement des pts. Ils possèdent habituellement deux PRD qui contiennent deux histidines conservées. Ces histidines sont des cibles potentielles de phosphorylations réversibles catalysées par le PTS (Figure 5). La plupart des protéines contenant des PRD sont activées via la phosphorylation catalysée par P-His-HPr au niveau d'une de ces histidines conservées. Pendant l'incorporation d'une source de carbone facilement métabolisable, telle que le glucose ou le fructose, HPr est déphosphorylée au niveau de son His-15. Elle ne phosphoryle donc plus les facteurs transcriptionnels contenant des PRD qui sont alors inactifs. $\varepsilon n$ revanche, lorsque ces facteurs transcriptionnels sont phosphorylés par des composants عIIA ou EIIB d'un PTS spécifique d'un sucre donné, ils sont inhibés; la transcription des gènes qu'ils régulent n'est donc pas activée. La déphosphorylation des composants EIIA ou EIIB pendant le transport et la phosphorylation du sucre concerné conduit également à la déphosphorylation du régulateur transcriptionnel contenant des PRD et donc à son activation (Figure 5). Les régulateurs transcriptionnels contenant des PRD participent donc à un mécanisme de RC qui est indépendant des protéines CcpA ou CRP [14, 33].

\section{Le rôle du PTS dans l'exclusion de l'inducteur}

Chez de nombreuses bactéries, des substrats-PTS facilement métabolisables, comme le glucose, inhibent l'activité de transporteurs non-PTS impliqués dans l'incorporation de sources de carbone nonpréférentielles. Ce phénomène est appelé exclusion de l'inducteur [34]. Ces transporteurs, cibles de l'ElIAAlc chez les entérobactéries, ou de P-Ser-HPr chez les firmicutes, appartiennent souvent à la famille des transporteurs possédant une cassette de fixation d'ATP, plus communément appelés transporteurs $A B C$ (ou ATP-binding cassette transporters). Lors de ce processus d'exclusion de l'inducteur, le PTS, via les protéines $\varepsilon I I A^{G l c}$ ou HPr, exerce un effet indirect sur l'expression de gènes impliqués dans le transport et le catabolisme d'hydrates de carbone, en inhibant l'incorporation ou la synthèse de la molécule inductrice correspondante. Un exemple bien décrit chez plusieurs bactéries est le transporteur $A B C$ impliqué dans l'incorporation du maltose. Chez les entérobactéries Salmonella enterica serovar Typhimurium et $\varepsilon$. coli, lorsqu'un sucre rapidement métabolisable tel que le glucose est disponible, l'EIIA ${ }^{G l c}$ est présente principalement sous sa forme non phosphorylée [15]. Elle interagit spécifiquement avec le transporteur maltose et inhibe son activité (Figure 3). Des études structurales ont montré qu'un dimère d'EIIA ${ }^{G l c}$ non phosphorylée interagit avec un dimère de la protéine fixant les nucléotides (NBP), MalK, une des protéines constituant le transporteur $A B C$. Cette interaction arrête le cycle de transport du disaccharide en inhibant la fermeture ATP-dépendante du dimère de MalK $[35,36]$. Chez le firmicute Lactobacillus casei, des données génétiques suggèrent que c'est P-Ser-HPr qui inhibe le transporteur $A B C$ impliqué dans l'incorporation de maltose et de maltodextrose [36]. En effet, des mutants incapables de phosphoryler la Ser-46 de l'HPr ne présentent pas d'exclusion du maltose malgré la présence de glucose, alors qu'un mutant produisant constamment de la P-Ser-HPr n'est pas capable de transporter le maltose, même en l'absence de glucose. Il est à noter que, chez l'actinobactérie Corynebacterium glutamicum, le métabolisme du maltose est fortement altéré chez un mutant $p t s H$, dont le gène codant la protéine $\mathrm{HPr}$ a été délété, bien que le disaccharide soit incorporé via le transporteur $A B C$. En fait, la délétion de $p t s H$ s'avère inhiber spécifiquement l'expression du gène malP qui code la maltose phosphorylase [37]. Toutefois, les mécanismes moléculaires de cette régulation ne sont pas encore connus.

\section{Conclusion}

La $R C$ est un phénomène de régulation globale mis en place pour éviter la synthèse de protéines impliquées ou non dans le métabolisme carboné, et dont la présence n'est pas requise en présence de glucose (ou d'un hydrate de carbone facilement métabolisable). Par exemple, chez Vibrionaceae, le complexe AMPc-CRP régule plusieurs processus cellulaires, tels que la compétence naturelle ou la colonisation de l'animal hôte [19]. Chez L. monocytogenes, l'utilisation du glucose inhibe l'expression des gènes de virulence. Le mécanisme moléculaire impliqué n'est pas encore caractérisé, mais il est probable qu'un composant PTS y participe [38]. La $\mathrm{RC}$ met donc en jeu une variété de mécanismes moléculaires qui peuvent être spécifiques de l'espèce bactérienne. Bien souvent ces mécanismes font intervenir un composant du PTS, mais il existe quelques exceptions. Par exemple, chez les Streptomyces, le mécanisme moléculaire impliqué dans la RC, bien qu'encore inconnu, semble indépendant du PTS [39].

L'utilisation de sources de carbone préférentielles est un facteur déterminant dans le taux de croissance bactérienne [5]. Or, l'impact de la RC sur la composition d'une population bactérienne telle que le microbiote intestinal, qui peut comporter jusqu'à 500 espèces bactériennes cultivables, est encore très mal connu. II est clair qu'en fonction du régime alimentaire de l'hôte, la RC doit probablement avoir un rôle important dans la composition et la stabilité de ce microbiote [40, 41]. Dans les années à venir, l'étude des mécanismes moléculaires impliqués dans la RC chez des bactéries constituant un microbiote, mais aussi chez des espèces 
bactériennes nouvelles ou chez des pathogènes, ainsi que la détermination de l'impact de cette RC, tant du point de vue des bactéries que de celui de l'hôte, sont clairement des champs de recherche à explorer. $\diamond$

\section{SUMMARY}

Carbon catabolite repression or how bacteria choose their favorite sugars

Carbon catabolite repression is an important regulatory mechanism allowing bacteria, but also yeast and fungi, to preferentially use easily metabolizable carbon sources (like glucose) over relatively less favorable carbon sources (for example, organic acids and alcohols). This phenomenon is illustrated by diauxic growth during which bacteria assimilate firstly energy-efficient and rapidly metabolizable sugars then less-favored carbohydrates. A variety of molecular mechanisms are involved in carbon catabolite repression in order to control not only the expression of genes involved in the utilization of alternative carbon sources but also the expression of genes involved in several processes like virulence, competence etc. In this review, are described the main molecular mechanisms found in enterobacteria and in firmicutes and the importance of the sugar-uptake phosphotransferase system for these molecular mechanisms. $\diamond$

\section{REMERCIEMENTS}

Ce travail a été financé par le CNRS et l'Université d'Aix-Marseille. Je remercie chaleureusement J.R. Fantino pour son aide dans la réalisation des figures et $F$. Pompeo pour ses suggestions et sa lecture critique du manuscrit.

\section{LIENS D'INTÉRÊT}

L'auteure déclare n'avoir aucun lien d'intérêt concernant les données publiées dans cet article.

\section{RéFÉRENCES}

1. Monod J. Recherches sur la croissance des cultures bactériennes. Thèse PhD, Institut Pasteur, Paris, France, 1942

2. Van der Stel AX, van de Lest CHA, Huynh S, et al. Catabolite repression in Campylobacter jejuni correlates with intracellular succinate levels. Environ Microbiol $2018 ; 20$ : 1374-88.

3. Poncet $S$, Milohanic $\varepsilon$, Mazé $A$, et al. Correlations between carbon metabolism and virulence in bacteria. Contrib Microbiol $2009 ; 16: 88-102$.

4. Moreno MS, Schneider BL, Maile RR, et al. Catabolite repression mediated by the CcpA protein in Bacillus subtilis: novel modes of regulation revealed by whole-genome analyses. Mol Microbiol $2001 ; 39$ : 1366-81.

5. Görke B, Stülke J. Carbon catabolite repression in bacteria: many ways to make the most out of nutrients. Nat Rev Microbiol 2008 ; 6 : 613-24.

6. Rojo F. Carbon catabolite repression in Pseudomonas: optimizing metabolic versatility and interactions with the environment. FEMS Microbiol Rev 2010 ; 34 : 658-84.

7. Wang $X$, Goh $\varepsilon B$, Beller HR. Engineering $\varepsilon$. coli for simultaneous glucose-xylose utilization during methyl ketone production. Microb Cell Fact $2018 ; 17: 12$.

8. Deutscher J. The mechanisms of carbon catabolite repression in bacteria. Curr Opin Microbiol $2008 ; 11: 87-93$.

9. Hoffee P, Englesberg $\varepsilon$, Lamy F. The glucose permease system in bacteria. Biochim Biophys Acta $1964 ; 79: 337-50$.

10. Kundig W, Ghosh S, Roseman S. Phosphate bound to histidine in a protein as an intermediate in a novel phospho-transferase system. Proc Natl Acad Sci USA 1964 ; 52 : 1067-74.

11. Postma PW, Lengeler JW, Jacobson GR. Phosphoenolpyruvate:carbohydrate phosphotransferase systems of bacteria. Microbiol Rev 1993 ; 57 : 543-94.

12. Reizer J, Deutscher J, Saier MH. Metabolite-sensitive, ATP-dependent, protein kinase-catalyzed phosphorylation of $\mathrm{HPr}$, a phosphocarrier protein of the phosphotransferase system in grampositive bacteria. Biochimie $1989 ; 71: 989-96$.

13. Galinier A, Kravanja M, Engelmann R, et al. New protein kinase and protein phosphatase families mediate signal transduction in bacterial catabolite repression. Proc Natl Acad Sci USA 1998 ; 95 : 1823-8.
14. Galinier A, Deutscher J. Sophisticated regulation of transcriptional factors by the bacterial phosphoenolpyruvate: sugar phosphotransferase system. J Mol Biol 2017 ; 429 : 773-89.

15. Bettenbrock K, Sauter T, Jahreis K, et al. Correlation between growth rates, EIIA ${ }^{\text {Crr }}$ phosphorylation, and intracellular cyclic AMP levels in Escherichia coli K-12. J Bacteriol $2007 ; 189: 6891-900$.

16. Amin N, Peterkofsky A. A dual mechanism for regulating cAMP levels in Escherichia coli. J Biol Chem 1995 ; 270 : 11803-5.

17. Eron L, Arditti R, Zubay G, et al. An adenosine 3':5'-cyclic monophosphatebinding protein that acts on the transcription process. Proc Natl Acad Sci USA $1971 ; 68: 215-8$.

18. Brückner R, Titgemeyer F. Carbon catabolite repression in bacteria: choice of the carbon source and autoregulatory limitation of sugar utilization. FEMS Microbiol Lett $2002 ; 209$ : 141-8.

19. Colton DM, Stabb EV. Rethinking the roles of CRP, CAMP, and sugar-mediated global regulation in the Vibrionaceae. Curr Genet 2016; 62 : 39-45.

20. Henkin TM, Grundy FJ, Nicholson WL, Chambliss GH. Catabolite repression of alpha-amylase gene expression in Bacillus subtilis involves a trans-acting gene product homologous to the Escherichia coli Lacl and GalR repressors. Mol Microbiol $1991 ; 5: 575-84$.

21. Deutscher J, Küster $\varepsilon$, Bergstedt U, et al. Protein kinase-dependent HPr/ C cpA interaction links glycolytic activity to carbon catabolite repression in gram-positive bacteria. Mol Microbiol 1995 ; 15 : 1049-53.

22. Fujita Y, Miwa Y, Galinier A, Deutscher J. Specific recognition of the Bacillus subtilis gnt cis-acting catabolite-responsive element by a protein complex formed between CcpA and seryl-phosphorylated HPr. Mol Microbiol 1995 ; $17: 953-60$.

23. Jault JM, Fieulaine S, Nessler $S$, et al. The HPr kinase from Bacillus subtilis is a homo-oligomeric enzyme which exhibits strong positive cooperativity for nucleotide and fructose 1,6-bisphosphate binding. J Biol Chem 2000; 275 : 1773-80.

24. Kravanja M, Engelmann R, Dossonnet V, et al. The hprK gene of Enterococcus faecalis encodes a novel bifunctional enzyme: the HPr kinase/phosphatase. Mol Microbiol 1999 ; 31 : 59-66.

25. Hueck CJ, Hillen W. Catabolite repression in Bacillus subtilis: a global regulatory mechanism for the gram-positive bacteria? Mol Microbiol 1995 ; $15: 395-401$.

26. Miwa $Y$, Nakata A, Ogiwara A, et al. Evaluation and characterization of catabolite-responsive elements (cre) of Bacillus subtilis. Nucleic Acids Res $2000 ; 28: 1206-10$.

27. Marciniak $B C$, Pabijaniak $M$, de Jong $A$, et al. High- and low-affinity cre boxes for CcpA binding in Bacillus subtilis revealed by genome-wide analysis. BMC Genomics 2012; $13: 401$.

28. Galinier A, Haiech J, Kilhoffer MC, et al. The Bacillus subtilis crh gene encodes a HPr-like protein involved in carbon catabolite repression. Proc Natl Acad Sci USA 1997 ; $94: 8439-44$.

29. Landmann JJ, Werner S, Hillen W, et al. Carbon source control of the phosphorylation state of the Bacillus subtilis carbon-flux regulator $\mathrm{Crh}$ in vivo. FEMS Microbiol Lett $2012 ; 327: 47-53$.

30. Galinier A, Deutscher J, Martin-Verstraete I. Phosphorylation of either crh or HPr mediates binding of CcpA to the Bacillus subtilis xyn cre and catabolite repression of the xyn operon. J Mol Biol $1999 ; 286$ : 307-14.

31. Martin-Verstraete I, Deutscher J, Galinier A. Phosphorylation of HPr and Crh by HprK, early steps in the catabolite repression signalling pathway for the Bacillus subtilis levanase operon. J Bacteriol 1999; 181 : 2966-9.

32. Stülke J, Arnaud M, Rapoport G, Martin-Verstraete I. PRD: a protein domain involved in PTS-dependent induction and carbon catabolite repression of catabolic operons in bacteria. Mol Microbiol 1998 ; 28 : 865-74.

33. Deutscher J, Aké FM, Derkaoui M, et al. The bacterial phosphoenolpyru vate:carbohydrate phosphotransferase system: regulation by protein phosphorylation and phosphorylation-dependent protein-protein interactions. Microbiol Mol Biol Rev 2014 ; 78 : 231-56.

34. Lengeler JW, Jahreis K. Bacterial PEP-dependent carbohydrate: phosphotransferase systems couple sensing and global control mechanisms. Contrib Microbiol $2009 ; 16: 65-87$.

35. Wuttge S, Licht A, Timachi MH, et al. Mode of interaction of the signaltransducing protein $\varepsilon I I A^{G l c}$ with the maltose $A B C$ transporter in the process of inducer exclusion. Biochemistry $2016 ; 55: 5442-52$.

36. Monedero V, Yebra MJ, Poncet S, Deutscher J. Maltose transport in Lactobacillus casei and its regulation by inducer exclusion. Res Microbiol 2008 ; 159 : 94-102. 


\section{RéFÉRENCES}

37. Kuhlmann N, Petrov DP, Henrich AW, et al. Transcription of malP is subject to phosphotransferase system-dependent regulation in Corynebacterium glutamicum. Microbiology $2015 ; 161: 1830-43$

38. Aké FM, Joyet P, Deutscher J, Milohanic $\varepsilon$. Mutational analysis of glucose transport regulation and glucose-mediated virulence gene repression in Listeria monocytogenes. Mol Microbiol 2011 ; 81 : 274-93.

39. Romero-Rodríguez A, Rocha D, Ruiz-Villafán B, et al. Carbon catabolite regulation in Streptomyces: new insights and lessons learned. World J Microbiol Biotechnol $2017 ; 33: 162$
40. Tuncil Yદ, Xiao Y, Porter NT, et al. Reciprocal prioritization to dietary glycans by gut bacteria in a competitive environment promotes stable coexistence. MBio $2017 ; 8$.

41. Cao Y, Förstner KU, Vogel J, Smith CJ. cis-Encoded small RNAs, a conserved mechanism for repression of polysaccharide utilization in bacteroides.J Bacteriol 2016 ; $198: 2410-8$.

\section{TIRÉS À PART}

A. Galinier

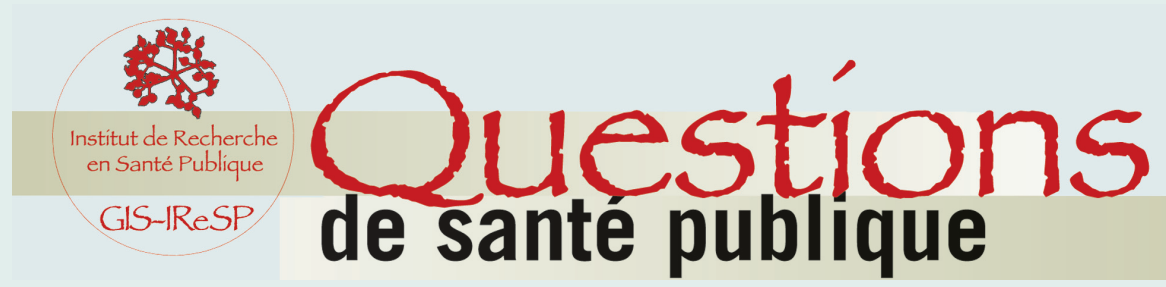

\section{Un nouveau bulletin

\author{
pour une meilleure \\ visibilité des résultats \\ de la recherche \\ en santé publique
}

es résultats de la recherche en santé publique souffrent en France d'un réel manque de visibilité. Ceci concerne aussi bien le monde académique (hors santé publique) que le grand public et les décideurs. Pour pallier ce déficit, I'IReSP a créé un bulletin à large diffusion intitulé "Questions de santé publique ", largement inspiré du bulletin mensuel d'information de I'INED « Populations et sociétés ". L'objectif éditorial est de porter à la connaissance d'un large public (enseignants, étudiants, journalistes, décideurs, milieux de la recherche, asso- ciations, public concerné) les informations les plus récentes concernant des questions importantes de santé publique, rédigées de façon facilement lisible et compréhensible pour des non spécialistes, en garantissant que les informations publiées sont validées scientifiquement. La publication concerne des faits et non des positions. Au-delà de la présentation de résultats, les qualités pédagogiques de Questions de santé publique permettent au lecteur de mieux comprendre comment sont formulées et abordées les questions de santé publique et quelles sont les limites de ces études.

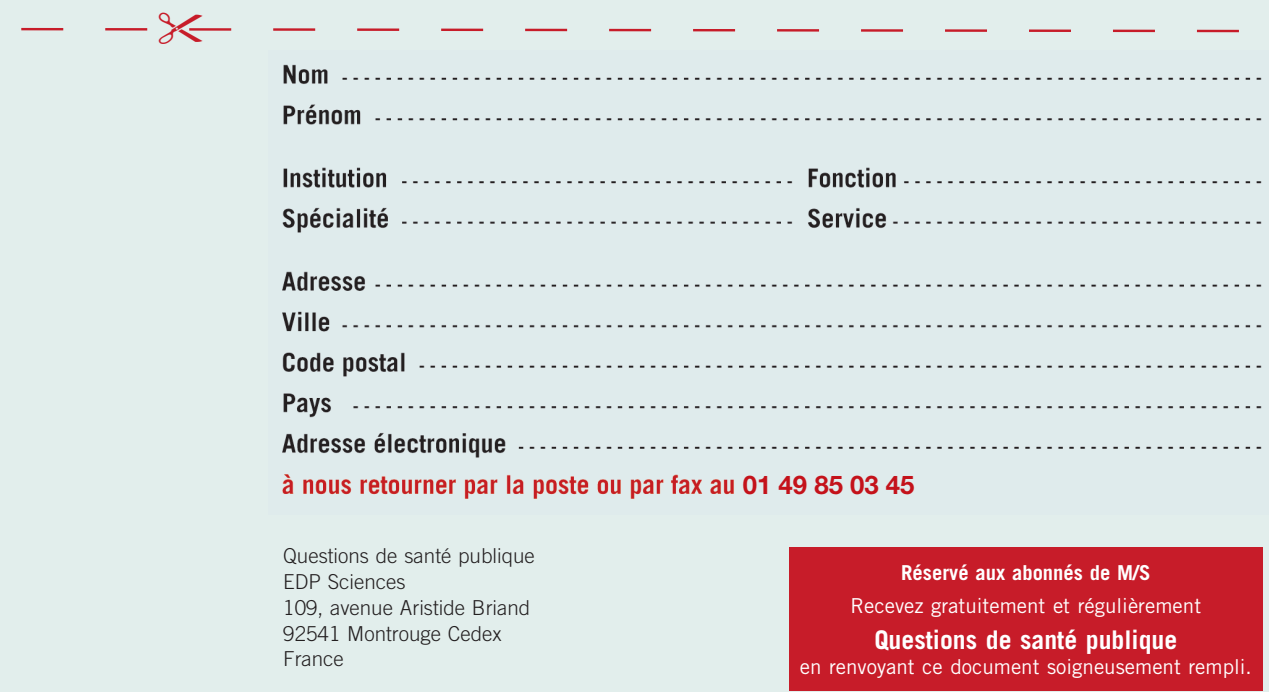

Questions de santé publique est une publication de l'Institut de Recherche en Santé Publique. I Directeur de la publication : Corinne Alberti. I Comité de rédaction : Sarah Bellouze, Marion Cipriano, Alexandre Cobigo et Jean-Marie Gagliolo. I Réalisation : EDP Sciences. 